\title{
Synovial fluid neutrophils of patients with rheumatoid arthritis have membrane antigen changes that reflect activation
}

\author{
PAUL EMERY, ${ }^{1}$ ANGEL F LOPEZ, ${ }^{2}$ GORDON F BURNS, ${ }^{2}$ AND \\ MATHEW A VADAS
}

From the ${ }^{1}$ Reid Memorial Laboratory of the Clinical Research Unit, The Walter and Eliza Hall Institute Medical Research, and the Royal Melbourne Hospital, Parkville, Victoria 3050; and the ${ }^{2}$ Division of Humain Immunology, Institute for Medical and Veterinary Science, Adelaide, South Australia 5000

SUMmary The level of expression of surface antigens was studied on neutrophils from pairee samples of blood and synovial fluid of patients with rheumatoid arthritis (RA). By measuring the immunofluorescence of labelled monoclonal antibodies it was shown that on joint neutrophil there was an increase in expression of complement receptor 3 (CR3) and granulocyte functiona antigens 1 and 2 compared with blood neutrophils, whereas the expression of GpIIb-IIIa wass reduced. The pattern of expression was the same as that seen with in vitro activation and strongto suggests that activation is occurring within the joint.

Key words: activation markers, monoclonal antibodies.

An understanding of the regulation of human neutrophil function has been enhanced by two sets of observations. Firstly, neutrophils are capable of being activated by naturally occurring cytokines such as colony stimulating factors ${ }^{1}$ and tumour necrosis factor. ${ }^{2}$ It has been suggested that these cytokines are responsible for the activation of neutrophils in vivo, especially at sites of inflammation such as that of RA. ${ }^{3}$ Additionally, neutrophils are activated by complement component $\mathrm{C} 5 \mathrm{a},{ }^{4}$ and a C5a related chemotactic activity has been demonstrated in synovial fluid (SF) from patients with RA. ${ }^{5}$ Secondly, the functional status of neutrophils is reflected by changes in surface antigens. The best studied example is CR3 recognised by various monoclonal antibodies (MAb) such as Mo-1. ${ }^{6}$ The congenital absence of this antigen leads to overwhelming sepsis in vivo and an absence of adherence mediated function in vitro, ${ }^{7}$ an effect which can be reproduced by certain anti-CR3 MAb. The concept that measurement of the level of antigen expression may reflect activation is supported by findings in vitro, where activation of neutrophils by bacterial

Accepted for publication 10 July 1987.

Correspondence to Dr Paul Emery, Rheumatology Unit, Guy's Hospital, London SE1 9RT. chemotactic agents such as f-Met-Leu-Phe (FMLP results in an enhancement of CR3 numbers on tho cell membrane,$^{68}$ and in vivo, where patients witb complement activation such as that seen aftew cupriphane membrane dialysis have activate neutrophils with enhanced CR3 expression. ${ }^{9}$

A second set of neutrophil restricted surface antigens, termed granulocyte functional antigens (GFA) 1 and 2, have been identified and MAQ against these structures augment several neutroph functions, including degranulation and antibod dependent, cell mediated cytotoxicity. ${ }^{10}$ These antigens are also enhanced by FMLP and colon stimulating factors, ${ }^{10}$ and thus their level of expreso sion may also reflect activation. The most recentfy described neutrophil antigen belongs to the GpIItos IIIa complex, a protein structure that serves as @ receptor for fibrinogen and fibronectin and was originally described on platelets. ${ }^{11}$ This antigen behaves differently from the above examples as ir expression is not increased on activation with FML (Lopez and Burns, unpublished observations).

The above observations strongly suggest tho neutrophils activated in vivo may express a surface phenotype reflecting their functional state, an $\$$ furthermore, that this phenotype may give ag independent measurement of the activity of some 
inflammatory disorders. In this communication we show that neutrophils purified from the joints of patients with active rheumatoid synovitis express significantly greater amounts of CR3 GFA-1 and GFA-2 antigen but less GpIIb-IIIa antigen than blood neutrophils from the same individual, isolated identically. Thus measurement of antigen expression on neutrophils may provide an independent assessment of the inflammatory response.

\section{Patients and methods}

\section{PATIENTS}

Thirteen patients (eight female, five male) attending the Royal Melbourne Hospital rheumatology clinic were studied. All had definite or classical seropositive rheumatoid arthritis (American Rheumatism Association criteria) of mean duration 16 years (range 2-45 years) and were receiving treatment which, in all but four cases, included disease modifying agents in addition to non-steroidal antiinflammatory drugs. No patient had received intraarticular steroids within the previous six months. Arthrocentesis was performed for diagnostic or therapeutic purposes and informed consent was obtained in accordance with an approved protocol. In one case (patient 13) both knees were aspirated. Blood was taken from controls who were healthy laboratory personnel.

\section{MEDIA AND REAGENTS}

The following media were used: Hanks's medium without $\mathrm{Ca}^{++}$or $\mathrm{Mg}^{++}$(Commonwealth Serum Laboratories, Melbourne, Australia), RPMI 1640
(Gibco, Chagrin Falls, OH, USA) plus $\mathrm{NaHCO}_{3}$, $\mathrm{NaCl}$, sodium pyruvate, penicillin, and streptomycin. HEPES buffered balanced salt solution ( $N-2-$ hydroxyethylpiperazine- $N^{\prime}$-2-ethanesulphonic acid), pH 7.2, was used for washing cells after incubation with MAb. Cells were fixed with $1 \%$ formaldehyde/ $5 \mathrm{mM} \mathrm{NaN} / 2 \%$ glucose in phosphate buffered saline. Fetal calf serum (FCS) (CSL, Melbourne) was heat inactivated for $30 \mathrm{~min}$ at $56^{\circ} \mathrm{C}$ and used at a concentration of $2 \%(\mathrm{v} / \mathrm{v})$. The chemotactic peptide FMLP was obtained from Sigma (St Louis, MO, USA).

\section{NEUTROPHIL SEPARATION}

Blood and SF were collected into Vacutainers containing heparin, free from preservative (BectonDickinson, Rutherford, NJ, USA). Dextran 7500 (Pharmacia, Uppsala Sweden) $(3 \%$ in normal saline) was added to the fluids in the ratio $1: 4$ dextran:fluid and left to stand for $45 \mathrm{~min}$. The leucocyte rich supernatant was then aspirated, washed in Hanks's medium and separated on FicollPaque (Pharmacia Piscataway, NJ, USA) at room temperature. The residual red blood cells in the pellet were lysed by osmotic shock using ice cold $0.2 \% \mathrm{NaCl}$ for $30 \mathrm{~s}$, followed by the addition of an equal volume of ice cold $1.6 \% \mathrm{NaCl}$, and the neutrophils were washed three times in RPMI/FCS. Cells were maintained at $4^{\circ} \mathrm{C}$ except for the period of density centrifugation. In stimulation experiments the effect of incubating cells at $37^{\circ} \mathrm{C}$ for 30 min with FMLP at a final concentration of $0 \cdot 1 \mu \mathrm{mol} / 1$ was tested, while other cells maintained at $37^{\circ} \mathrm{C}$ served as untreated controls. Finally, the cells were

Table 1 Antigen expression on peripheral blood (PB) and synovial fluid (SF) neutrophils of 13 patients with RA

\begin{tabular}{|c|c|c|c|c|c|c|c|c|c|c|c|c|c|c|}
\hline \multirow{3}{*}{$\begin{array}{l}\text { Patient } \\
\text { No }\end{array}$} & \multicolumn{14}{|c|}{ Antigen expression (mean fluorescence) } \\
\hline & \multicolumn{4}{|c|}{$C R 3$} & \multicolumn{4}{|c|}{ GpIIb-IIIa } & \multicolumn{4}{|c|}{$G F A-1$} & \multicolumn{2}{|c|}{$G F A-2$} \\
\hline & $P B$ & & $S F$ & & $P B$ & & $S F$ & & $P B$ & & $S F$ & & $P B$ & $S F$ \\
\hline 1 & 149 & $(6)^{*}$ & 334 & $(4)^{+}$ & 150 & (71) & 11 & (6) & 463 & $(12)$ & 524 & (10) & 51 & 91 \\
\hline 2 & 16 & (5) & 16 & $(8)^{+}$ & 10 & (4) & 4 & (4) & 131 & (5) & 161 & (3) & 100 & 165 \\
\hline 3 & 21 & (4) & 44 & $(5)^{+}$ & 126 & (11) & 11 & (11) & 230 & (5) & 439 & (7) & 23 & 115 \\
\hline 4 & 79 & (6) & 124 & $(3)^{+}$ & 16 & (5) & 11 & (5) & 106 & (4) & 110 & (8) & 40 & 96 \\
\hline 5 & 100 & (7) & 89 & $(7)^{+}$ & 23 & (6) & 5 & (5) & 4 & (5) & 128 & (8) & 12 & 15 \\
\hline 6 & 338 & (11) & 618 & (11) & 28 & (4) & 4 & (4) & 118 & (4) & 182 & (4) & 10 & 29 \\
\hline 7 & 529 & (20) & 524 & (28) & 82 & (22) & 44 & (24) & 129 & (20) & 604 & (28) & 139 & 166 \\
\hline 8 & 104 & (8) & 120 & (6) & 72 & (8) & 8 & (8) & 77 & (9) & 147 & (7) & 163 & 420 \\
\hline 9 & 104 & (4) & 127 & (4) & 92 & (9) & 14 & (11) & 116 & (11) & 162 & (18) & 54 & 56 \\
\hline 10 & 489 & (15) & 970 & (22) & 10 & (8) & 9 & (8) & 14 & (4) & 59 & (4) & 35 & 111 \\
\hline 11 & 162 & (20) & 328 & (28) & 11 & (10) & 10 & (14) & 25 & (14) & 31 & (21) & 58 & 92 \\
\hline 12 & 142 & (9) & 266 & (10) & 12 & (8) & 12 & (9) & 10 & (9) & 15 & (7) & 17 & 58 \\
\hline $13 \mathrm{~L}$ & 182 & (9) & 164 & (8) & 177 & (11) & 10 & (8) & 99 & (12) & 100 & (13) & 31 & 33 \\
\hline $13 \mathrm{R}$ & 182 & (12) & 199 & (13) & 177 & (11) & 10 & (10) & 99 & (12) & 110 & (13) & 31 & 55 \\
\hline
\end{tabular}

${ }^{*}$ The binding of control MAb is shown in brackets. These were MsIgG1 (or PB10 where indicated ${ }^{+}$) for CR3 and GFA-2, K7 for GFA-1, and second layer sheep antimouse $F\left(a b^{\prime}\right)_{2}$ alone for GpIIb-IIIa. 


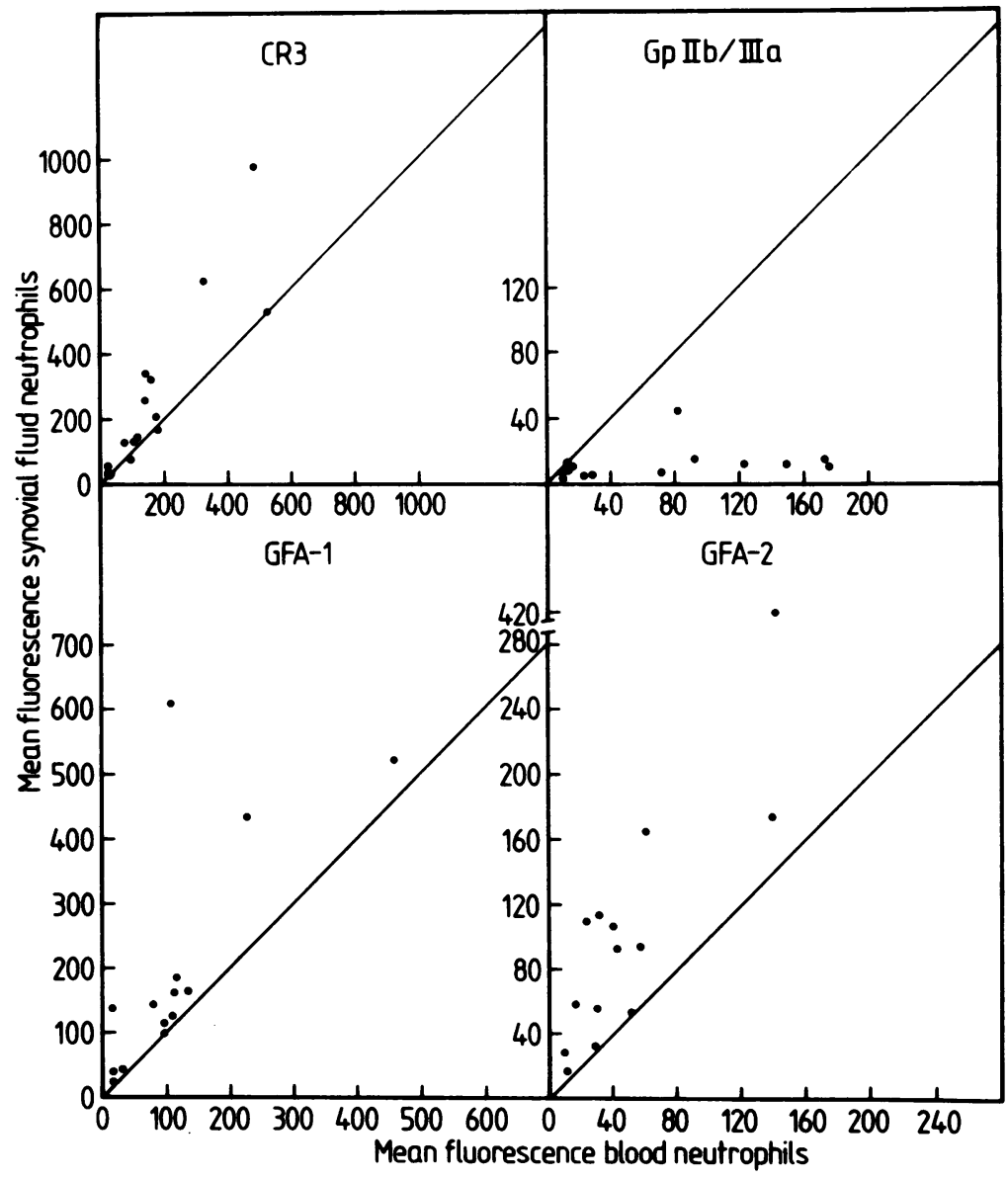

Fig. 1 Antigen expression (mea@ fluorescence) on neutrophils from peripheral blood or synovial fluid of 13 patients (14 samples) with rheumatoid arthritis. washed three times in RPMI/FCS and their concentration was readjusted to $10^{7}$ cells $/ \mathrm{ml}$.

MONOCLONAL ANTIBODIES

The following murine MAb were used: anti Mo-1 (IgG1) (Becton-Dickinson), WEM-G1 (IgM), WEM-G11 (IgG1), 25E11 (IgG2a), and anti- $\beta_{2}-\mathrm{m}$ (IgG2b). They recognise CR3, GFA-1, GFA-2, GpIIb-IIIa, and $\beta_{2}$ microglobulin respectively, and have been described previously. ${ }^{8}{ }^{9}$ In addition, the MAb K7 (IgM) (a gift of Dr G Klauss, National Institute for Medical Research, London) and PB10 (IgG1) (a gift of Dr P Bartlett, WEHI, Melbourne), which bind to the trinitrophenyl hapten and the chicken theta antigen respectively, together with MsIg (IgG1) (Becton-Dickinson) served as nonbinding, isotype matched controls. The MAb Hu-Pl- ml, which recognises an epitope on GpIIb-IIR whose expression is restricted to platelets, was a gif of Dr M Sandrin (Centre for Transplantation and Cancer Research, Melbourne). All the MAb wefe fluoresceinated, with the exception of the two antiGpIIb-IIIa MAb; in these cases a fluoresceinate $\mathrm{F}\left(\mathrm{ab}^{\prime}\right)_{2}$ sheep antimouse immunoglobulin was used as a second layer.

CELL STAINING AND FLOW CYTOMETRE A N A L Y S IS

Neutrophils $(50 \mu l)$ in microtitre wells were inc? bated with saturating concentrations of appropria $\mathrm{MAb}$ at $4^{\circ} \mathrm{C}$ for $45 \mathrm{~min}$. This was followed by three washes at $4^{\circ} \mathrm{C}$ in balanced salt solution and fixation before analysis by flow cytometry. For non-fluoe esceinated MAb, incubation with the second layer 
MAb, followed by a further three washes, was performed before fixation. Cells were analysed using a fluorescence activated cell sorter (FACS model II, Becton-Dickinson) and were gated by size and $90^{\circ}$ scatter to select for neutrophils.

STATISTICAL ANALYSIS

After conversion to a linear scale the mean fluorescence values relating to peripheral blood (PB) and SF neutrophil samples of individual subjects were

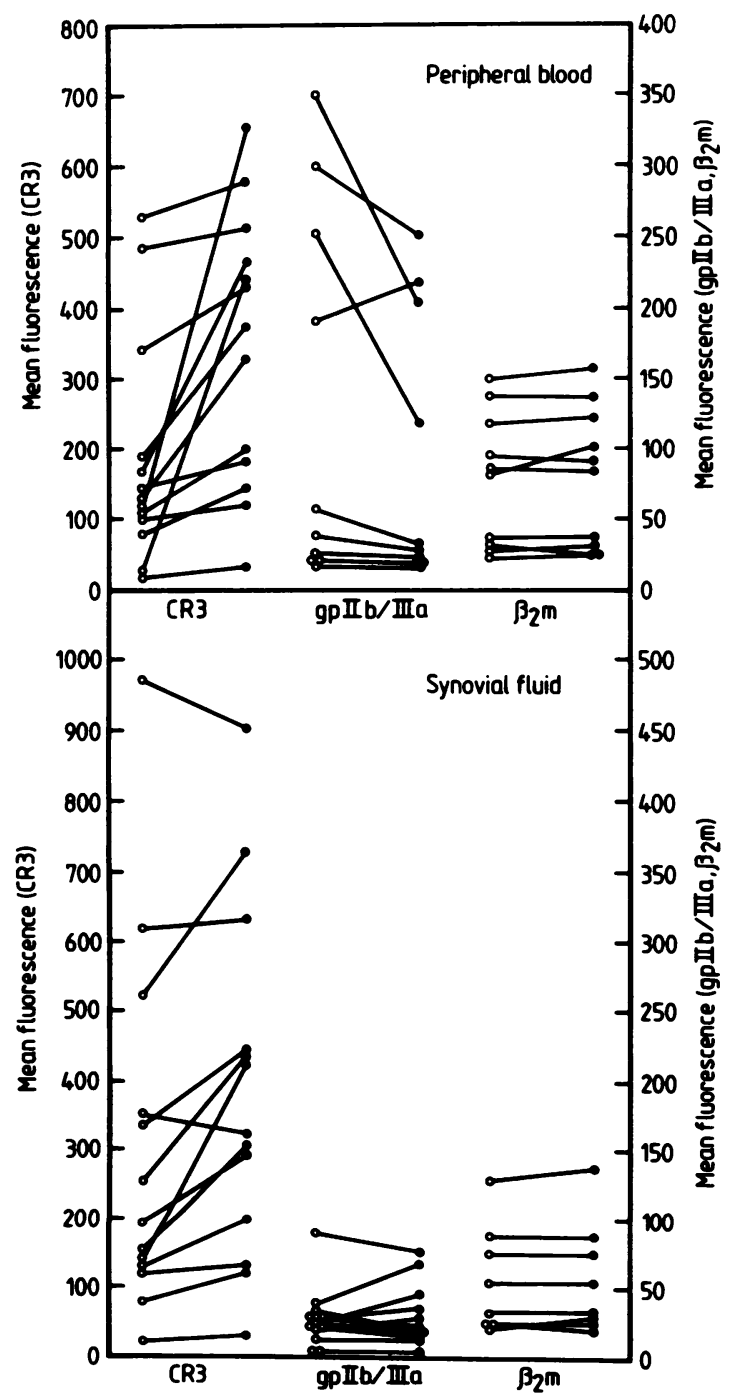

Fig. 2 Change in antigen expression (mean fluorescence) on peripheral blood or synovial fluid neutrophils stimulated with FMLP (O) in comparison with unstimulated cells $(\bigcirc)$. compared using the non-parametric Wilcoxon signed rank test.

\section{Results}

EXPRESSION OF ANTIGENS ON NEUTROPHILS FROM BLOOD AND SYNOVIAL FLUID OF PATIENTS WITH RA

The expression of CR3, GFA-1, and GFA-2 on synovial fluid neutrophils was greater than on neutrophils from peripheral blood (Table 1; $\mathrm{p}<0.003$ ); significantly increased antigen expression on SF neutrophils was seen in at least 10 samples for all of these antigens. In contrast, the expression of GpIIb-IIIa was reduced on SF neutrophils $(p<0.001)$. Plots of the relative expression of the antigens in blood versus synovial fluid illustrate these features (Fig. 1). The binding of $\mathrm{Hu}-\mathrm{Pl}-\mathrm{ml}$ was studied to assess specific binding to platelets and thus exclude the possibility that the binding of

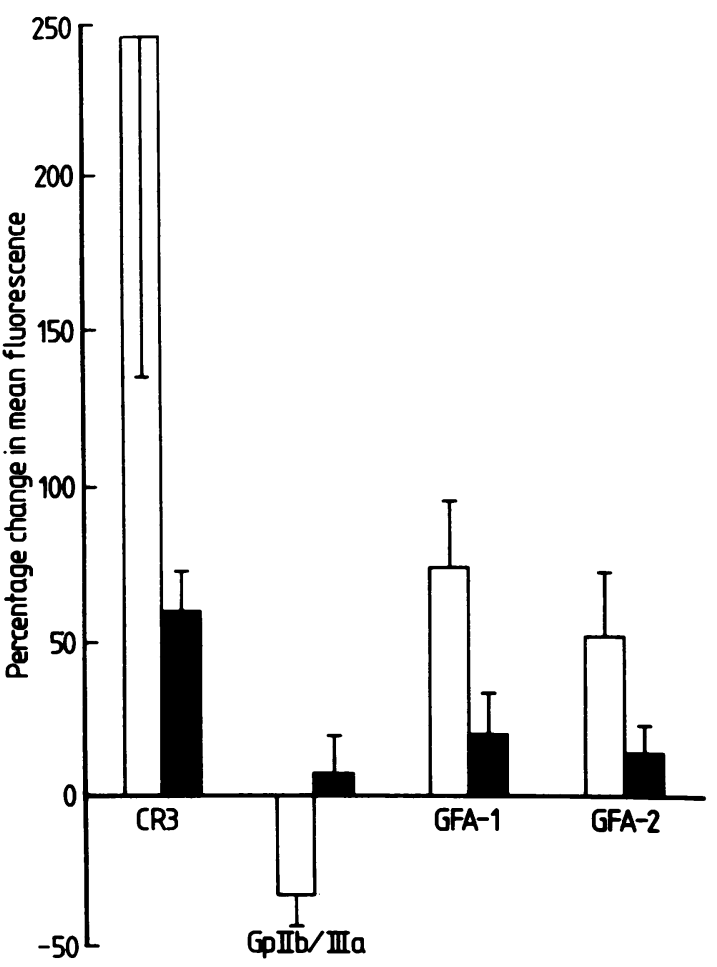

Fig. 3 Antigen expression on neutrophils from peripheral blood (white columns) or synovial fluid (black columns) after stimulation with FMLP. Results expressed as percentage change in mean antigen expression in comparison with unstimulated neutrophils from the same source. (The bars are one standard error.) 
$25 \mathrm{E} 11$ could be attributed to contamination of the neutrophil preparation by adherent platelets. In seven experiments there was no significant binding to any of the neutrophil preparations (data not shown).

EFFECT OF FMLP ON ANTIGEN EXPRESSION FMLP significantly enhanced the expression of CR3, GFA-1, and GFA-2 but reduced that of GpIIb-IIIa on PB neutrophils $(\mathrm{p}<0.02)$ (data shown for CR3 and GpIIb-IIIa in Fig. 2). The effect of FMLP on SF neutrophils was less consistent and ultimately not significant $(p>0.06)$, though the expression of CR3, GFA-1, and GFA-2 tended to be enhanced (Fig. 3).

\section{Discussion}

We have shown that in patients with RA neutrophils from SF have altered expression of surface molecules. Furthermore, this altered expression follows a pattern seen when neutrophils are activated in vitro, strongly suggesting that this activation is occurring in vivo.

The role of neutrophils in the pathogenesis of articular destruction in RA is unclear. These cells predominate in the SF, however, during acute exacerbations of the disease, are seen in the vicinity of synovial blood vessels or at the articular margin, and are known to secrete a number of pro-inflammatory mediators after stimulation in vitro. ${ }^{3}$ Studies comparing the functional activity of SF neutrophils with those from PB have yielded conflicting results. ${ }^{12-15}$ It has been noted that the phagocytic activity of SF neutrophils is depressed or enhanced, whereas no differences in resting enzyme content or stimulated enzyme release are apparent when differences in viability are taken into account. ${ }^{13}$ Potential explanations for these discrepancies include technical differences in isolation methods or assays and variable degrees of activation in vivo which could render the cells refractory to further stimulation in vitro. This was clarified by the use of antigen expression as an independent marker of function status.

Previous work in this laboratory has identified two antigens, GFA-1 and GFA-2, which are involved in antibody dependent killing, ${ }^{10}$ and a third, GpIIb-IIIa, the receptor for fibrinogen and fibronectin, which is involved in cell adhesion. ${ }^{910}$ As has been shown for CR1 and CR3 the expression of GFA-1 and GFA-2 is enhanced by stimuli which activate neutrophils ${ }^{10}$; this was confirmed in the present study, and it was also shown that the expression of GpIIb-IIIa is reduced. Therefore it seemed that the relative level of expression of these antigens would serve as a marker of the function馬 status of neutrophils from sites of inflammation such as synovial fluid. Preliminary investigations (da) not shown) have confirmed that the conditions of neutrophil isolation can affect the expression of surface antigens. ${ }^{16}$ Consequently, SF and PB neftrophils were prepared identically, and the pattefn of expression of several antigens was compare $\vec{\Phi}$ rather than relying on the absolute expression of any one antigen.

The results of the present study show that the expression of CR3, GFA-1, and GFA-2 is increaseg on SF neutrophils and that of GpIIb-IIIa is dimin ished compared with PB cells. This is in keeping wiţ the pattern of reactivity after stimulation in vitro and supports the hypothesis that the SF neutrophils have been activated in vivo. These findings are also consistent with enhanced CR3 expression on new trophils from sterile exudates ${ }^{17}$ and a recent demo stration of increased C3 receptors on SF neutrophi⿺s using a rosette assay. ${ }^{14}$ On the other hand, ouf failure to stimulate consistently further enhancee ment of these antigens on SF neutrophils by incubation with FMLP in vitro is not necessarily contrab to the finding of increased FMLP receptors an responsiveness after exudation ${ }^{17}$ as the nature and duration of the in vivo stimulus could not tse ascertained or controlled. The level of activation $\mathscr{f}$ PB neutrophils was not addressed in this stud $\overrightarrow{\overrightarrow{6}}$, firstly, because our prime interest was in expressio on SF neutrophils and, secondly, because cro吕 sectional analysis would have been hard to interpreit with wide ranges between individuals.

Our findings have several implications for the management of inflammatory disorders. Firstly, the monitoring of neutrophil activation antigens mà provide an independent assessment of the efficacy of anti-inflammatory treatment, and could ultimate $\vec{y}$ be a very useful test. As baselines differ between individuals these tests will need to be performea serially or, as we have in this communication, by comparing two sites in the body. Secondly, it possible that the pattern of antigenic changes may reflect the agent responsible for activation. We have already noted that activation mediated by tumour necrosis factor results in quantitatively and qualitatively different changes in these antigens, ${ }^{2}$ and it ${ }^{\circ}$ thus possible that in the future therapy could be tailored according to these parameters. Thirdly, the present findings clearly establish that neutrophit have an altered phenotype consistent with a state of activation of the cell in the synovium and suggest the possibility that the localised activation of neutro phils in the joint may play a part in tissue destruction observed in patients with RA. 
We thank Dr James Williamson for his constructive comments. PE was supported by grants from the National Health and Medical Research Council of Australia.

\section{References}

1 Lopez A F, Williamson D J, Gamble J R, et al. A recombinant human granulocyte-macrophage colony stimulating factor $(\mathrm{rH}$ GM-CSF) stimulates in vitro mature human neutrophil and eosinophil function, surface receptor expression and survival. $J$ Clin Invest 1986; 78: 1220-8.

2 Gamble J R, Harlan J M; Klebanoff S J, Vadas M A. Stimulation of the adherence of neutrophils to umbilical vein endothelium by human recombinant tumor necrosis factor. Proc Natl Acad Sci USA 1985; 82: 8667-71.

3 Weissman G, Sehman C, Korchak H M, Smolen J E. Neutrophils: release of mediators of inflammation with special reference to rheumatoid arthritis. Ann NY Acad Sci 1982; 389: 11-24.

4 Hugli T E, Muller-Eberhard H J. Anaphylotoxins C3a and C5a. Advan Immunol 1978; 26: 1-53.

5 Ward P A, Zvaifler N J. Complement-derived leukotaxic factors in inflammatory synovial fluids of humans. $J$ Clin Invest 1971; 50: 606-16.

6 Wright S D, Rao P E, Van Voorhis W C, et al. Identification of the C3bi receptor of human monocytes and macrophages using monoclonal antibodies. Proc Natl Acad Sci USA 1983; 80: 5699-703.

7 Anderson D C, Schmalsteig F C, Arnaout M A, et al. Abnormalities of polymorphonuclear leukocyte function associated with a heritable deficiency of high molecular weight surface glycoproteins (GP138): common relationship to diminished cell adherence. J Clin Invest 1984; 74: 536-51.

8 Berger M, O'Shea J, Cross A S, et al. Human neutrophils increase expression of $\mathrm{C} 3 \mathrm{bi}$ as well as $\mathrm{C} 3 \mathrm{~b}$ receptors upon activation. J Clin Invest 1984; 74: 1566-71.

9 Lee J, Hakim R M, Fearon D T. Increased expression of the $\mathrm{C} 3 \mathrm{~b}$ receptor by neutrophils and complement activation during haemodialysis. Clin Exp Immunol 1984; 56: 205-14.

10 Vadas M A, Lopez A F, Williamson D J. Selective enhancement of the expression of granulocyte functional antigen 1 and 2 on human neutrophils. Proc Natl Acad Sci USA 1985; 82: 2503-7.

11 Burns G F, Cosgrove L, Triglia T, et al. The IIb-IIIa glycoprotein complex that mediates platelet aggregation is directly implicated in leukocyte adhesion. Cell 1986; 45: 269-80.

12 Turner R A, Schumacher H R, Meyers A R. Phagocyte function of polymorphonuclear leucocytes in rheumatic diseases. J Clin Invest 1973; 52: 1632-5.

13 Van de Stadt R J, Van de Voorde Vissers E, Feltkamp-Vroom T M. Metabolic and secretory properties of peripheral and synovial granulocytes in rheumatoid arthritis. Arthritis Rheum 1980; 23: $17-23$.

14 Goddard D H, Kirk A P, Kirwan J R, Johnson G D, Holborow E J. Impaired polymorphonuclear leucocyte chemotaxis in rheumatoid arthritis. Ann Rheum Dis 1984; 43: 151-6.

15 Breedveld F C, Lafeber G J M, Van den Barselarr M T, Van Dissel J T, Leijh P C J. Phagocytosis and intracellular killing of Staphylococcus aureus by polymorphonuclear cells from synovial fluid of patients with rheumatoid arthritis. Arthritis Rheum 1986; 29: 166-73.

16 Fearon D T, Collins L A. Increased expression of C3b receptors on polymorphonuclear leukocytes induced by chemotactic factors and by purification procedures. J Immunol 1983; 130: 370-5.

17 Zimmerli W, Seligmann B, Gallin J I. Exudation primes human and guinea pig neutrophils for subsequent responsiveness to the chemotactic peptide $\mathrm{N}$-formylmethionylleucylophenylalanine and increases complement component $\mathrm{C} 3 \mathrm{bi}$ receptor expression. J Clin Invest 1986; 77: 925-33. 